\title{
Growth and Disappearance of Nanobubbles during the Foaming of Polycarbonate
}

\author{
By Yoshihito FuKASAWA, ${ }^{1}$ Hiromu SAITo, ${ }^{1, *}$ Motohisa KATO, ${ }^{2}{\text { Tsuneo CHIBA },{ }^{2} \text { and Takashi INOUE }}^{3}$
}

We investigated the structural development of bubbles during the foaming of polycarbonate under carbon dioxide $\left(\mathrm{CO}_{2}\right)$ by using a field emission scanning electron microscope (FE-SEM). Numerous $\mathrm{CO}_{2}$ nanopores having a size of around $50 \mathrm{~nm}$ were obtained by rapidly cooling the nanobubbles. At an early stage of the foaming, the size and number density of the nanobubbles increased over time. However, the number density of the bubbles later decreased and only a small number of nanobubbles grew to a larger size of micrometer scale, suggesting that most of the initially formed nanobubbles do not grow but disappear during the foaming. The disappearance of the nanobubbles might be attributed to the coalescence of neighboring nanobubbles and Ostwald ripening in which large bubbles grow at the expense of the small nanobubbles.

KEY WORDS: FE-SEM / Foam / Nano-bubble / Ostwald Ripening / Polycarbonate / Pore /

New phase formation is a common physical phenomenon for processes such as crystallization, solidification, boiling, phase separation of polymer blends, and foaming., ${ }^{1,2}$ Two major mechanisms are known to occur in new phase formation. ${ }^{3-6}$ One is spinodal decomposition in which a wavelike concentration fluctuation with periodic wavelength increases over time by uphill diffusion, producing a co-continuous structure. The other is nucleation and growth in which particles are nucleated and then grow spherically over time. In the theory of the process of nucleation and growth, a nucleated particle can grow spontaneously when the particle exceeds a critical size for growth while particles smaller than the critical size are inherently unstable and disappear. ${ }^{1,2,7-9}$ These concepts have been derived from the kinetic aspect of the growth of particles at the micrometer level suggested by scanning electron microscopy (SEM) and optical microscopy. So far, however, the structural development of nanometer-scale particles around a critical size has not been elucidated although it is helpful to completely understand the nucleation and growth process. Observation of nanometer-scale particles during nucleation and growth is expected to clarify the question as to whether all initially nucleated nanoparticles grow to larger ones of a micrometer scale, for instance.

In this study, to understand the nucleation and growth process of nanobubbles, we investigate the structural development of the bubbles at an early stage of the foaming of polycarbonate by using a field emission scanning electron microscope (FE-SEM). This FE-SEM has a resolution that is one hundred times higher than that of a conventional SEM and is powerful enough to characterize the structure at a nanometer level. The FE-SEM observation let us notice that initially nucleated nanobubbles could be frozen by rapid cooling of the foamed specimen. To clarify the characteristics of nanobubbles, the structural development of nanobubbles is com- pared with that of micrometer-scale bubbles as observed by an optical microscope.

\section{EXPERIMENTAL}

The polycarbonate plate specimen used in this study was supplied by General Electric Co., Ltd. (Lexan 9030).

The plate specimen with a thickness of $1.5 \mathrm{~mm}$ was placed in a 200-mL stainless steel pressure vessel (Taiatsu Techno TVS-N2). After the vessel was sealed, $\mathrm{CO}_{2}$ was injected from a cylinder until the pressure reached $0.5 \mathrm{MPa}$ and the specimen was saturated with $\mathrm{CO}_{2}$ at room temperature for $48 \mathrm{~h}$. Then, the specimen was depressurized to ambient pressure and removed from the vessel. The $\mathrm{CO}_{2}$-saturated specimen thus obtained was rapidly transferred to a hot stage set at $140{ }^{\circ} \mathrm{C}$, which is higher than the glass transition temperature of $\mathrm{CO}_{2}$-saturated polycarbonate. The structural development of the $\mathrm{CO}_{2}$-saturated polycarbonate during the isothermal annealing was observed under an optical microscope (Olympus, BX-50). The image data was stored in a personal computer.

After the isothermal annealing, the specimen was rapidly cooled in an ice water bath within 1 second to freeze the structure. The structure of the specimen thus obtained was observed under a Hitachi S-800 FE-SEM at an accelerating voltage of $10 \mathrm{kV}$. For FE-SEM observation, a thin section of the plate specimen was cut with a microtome. The thin section was sputter-coated with carbon to improve the conductivity of the surface.

\section{RESULTS AND DISCUSSION}

Figure 1 shows the observed in situ structure of the development of micrometer-scale bubbles by using the optical microscope during the annealing of the $\mathrm{CO}_{2}$-saturated poly-

\footnotetext{
${ }^{1}$ Department of Organic and Polymer Materials Chemistry, Tokyo University of Agriculture and Technology, Koganei 184-8588, Japan

${ }^{2}$ Department of Organic and Polymeric Materials, Tokyo Institute of Technology, 2-12-1 Ookayama, Meguro-ku, Tokyo 152-8552, Japan

${ }^{3}$ Department of Polymer Science and Technology, Yamagata University, Jonan, Yonezawa 992-8510, Japan

*To whom correspondence should be addressed (Tel \& Fax: +81-42-388-7294; E-mail: hsaitou@ @c.tuat.ac.jp).
} 




(a) $\mathrm{t}=12 \mathrm{~s}$



(b) $\mathrm{t}=18 \mathrm{~s}$
Figure 1. Structural development of bubbles during the annealing of $\mathrm{CO}_{2}$ saturated polycarbonate at $140^{\circ} \mathrm{C}$.

carbonate at $140^{\circ} \mathrm{C}$. No structure was seen and the specimen was transparent before the annealing time of $10 \mathrm{~s}$. Spherical bubbles having a diameter of several micrometers appeared at around $10 \mathrm{~s}$ and they grew to several tens of micrometers with increased annealing time. This indicates that foaming occurs and the bubbles grow as the $\mathrm{CO}_{2}$-saturated polycarbonate is annealed. Spherical pores were obtained by rapid cooling after the annealing, suggesting that the structure of the bubbles could be frozen by rapid cooling.

The distance between neighboring bubbles was about $100 \mu \mathrm{m}$; this distance did not change during the annealing. That is, the number density of the bubbles did not change during the foaming, suggesting that new bubbles did not nucleate on a micrometer scale during the foaming. The growth of the bubbles stopped before the impingement and coalescence of neighboring bubbles. Our interest is the structural development of the bubbles on a nanometer scale during the foaming, which is not observable under an optical microscope or conventional SEM. To elucidate the structural development of bubbles on a nanometer scale, FE-SEM observation of the nanobubbles was demonstrated as in the following example. For instance, can all initially nucleated bubbles grow to larger ones from a nanometer scale to a micrometer scale?

Figure 2 shows FE-SEM micrographs of pores obtained by freezing the bubbles developed during the foaming of the $\mathrm{CO}_{2}$ saturated polycarbonate at $140{ }^{\circ} \mathrm{C}$ for various annealing times. Here, the saturated pressure is low at $0.5 \mathrm{MPa}$ in which the growth rate of the bubble is slow and the nanobubbles can be frozen and obtained at an early stage of the foaming. Numerous pores are seen as white spheres (Figure 2a: $\mathrm{t}=5 \mathrm{~s}$ ) although the specimen was transparent and no structure was seen under an optical microscope. The pore diameter is $50 \mathrm{~nm}$. These results indicate that nanoporous transparent material is obtained by freezing the nanobubbles that evolved at an early stage of the foaming. The number of nanopores increased over time (Figure $2 \mathrm{~b}: \mathrm{t}=7 \mathrm{~s}$ ), suggesting that new nanobubbles are formed in the polycarbonate-rich matrix between preexisting bubbles during the foaming, although the number of bubbles does not increase on a micrometer scale, as demonstrated in Figure 1. Since the new nanobubbles are formed during the growth of preexisting bubbles, large pores coexisted with small

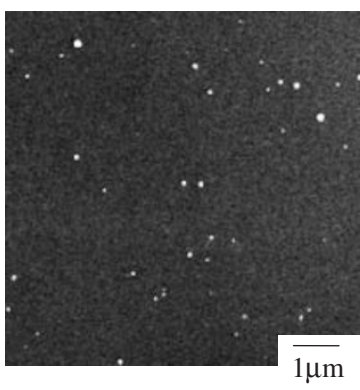

(a) $t=5 \mathrm{~s}$



(c) $t=9 \mathrm{~s}$



(b) $t=7 s$

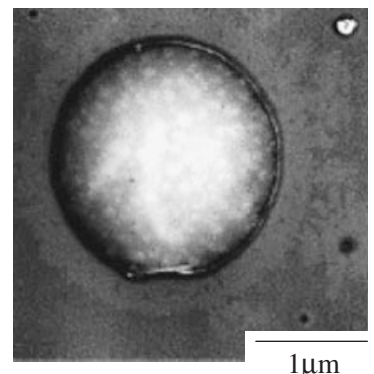

(d) $\mathrm{t}=11 \mathrm{~s}$
Figure 2. FE-SEM micrographs of pore structure of polycarbonate obtained after annealing the $\mathrm{CO}_{2}$-saturated specimen at $140^{\circ} \mathrm{C}$ for various times.

ones, and the size distribution of bubbles grew wider. The most interesting result here is that the distance between the neighboring nanobubbles is hundreds times shorter than that observed under an optical microscope (shown in Figure 1). That is, the distance between the nanopores is several hundred nanometers while it is a hundred micrometers for micrometerscale pores. The results suggest that the number of bubbles decreases extraordinarily during the foaming.

According to the theory of nucleation and growth, a nucleus can grow spontaneously to a larger one when its size exceeds the critical size $r^{*}$ while a nucleus smaller than $r^{*}$ is inherently unstable and disappears. The critical bubble size in diameter $r^{*}$ for a spherical nucleus is given by ${ }^{1,2,7-11}$

$$
r^{*}=4 \sigma / \Delta P
$$

where $\sigma$ is the surface tension of the polymer-bubble interface, which increases with decreasing gas pressure, and $\Delta P$ is the pressure difference between the bubble pressure and the ambient pressure. Since $\sigma$ of polymers at around $0.5 \mathrm{MPa}$ is about $3 \times 10^{-2} \mathrm{~N} / \mathrm{m}$ as demonstrated in Reference 11 and $\Delta P$ in this system is $0.5 \times 10^{6} \mathrm{~N} / \mathrm{m}^{2}, r^{*}$ is calculated as being about $240 \mathrm{~nm}$. As shown in Figure $2 \mathrm{a}$ and $2 \mathrm{~b}$, the diameter of the nanopores is less than $240 \mathrm{~nm}$. Thus, the results might imply that the nanobubbles initially formed before $7 \mathrm{~s}$ are smaller than the critical bubble size and are frozen by the rapid cooling.

Neighboring nanobubbles impinged on each other and then the impinged bubbles coalesced and the spherical bubbles changed to large deformed ones having a size of a hundred nanometers (Figure 2c: $\mathrm{t}=9 \mathrm{~s}$ ). The deformed bubbles then grew to large spherical ones having the size of a micrometer 




Figure 3. Number density of bubbles and average bubble diameter as a function of annealing time for $\mathrm{CO}_{2}$-saturated polycarbonate obtained after annealing at $140^{\circ} \mathrm{C}$.

(Figure $2 \mathrm{~d}: \mathrm{t}=11 \mathrm{~s}$ ). The size of the large spherical bubble is much larger than that of the distance between the neighboring nanobubbles. That is, the size of the large spherical bubble is several micrometers while the distance between the neighboring nanobubbles is several hundred nanometers. Thus, neighboring nanobubbles existing around the large bubbles do not grow larger, but disappear.

The number density of bubbles and average bubble diameter were obtained by FE-SEM observation and the results are shown in Figure 3 as a function of annealing time. Owing to the formation of new nanobubbles during the growth of preexisting bubbles, the number density of bubbles increases while the average bubble diameter decreases with annealing time at early stage of the foaming before $9 \mathrm{~s}$. The number density of bubbles does not monotonously increase with increasing annealing time but it decreases steeply after $12 \mathrm{~s}$ associating with the increase of the average bubble diameter of a few large bubbles. The results suggest that most of the initially formed nanobubbles disappear except for a few that grow larger. The disappearance of the nanobubbles might be attributed to Ostwald ripening. The large particles grow at the expense of the small ones by the diffusion of molecules from the smaller particles while the initially formed small particles disappear except for a few that grow larger because small particles act as a source for the growth of the larger particles. ${ }^{4,12-14}$

As shown in Figure 4a, the large spherical bubbles grew and the size became larger with annealing time to yield bubbles having a diameter of several tens of micrometers. Since the distance between the neighboring nanobubbles was several hundred nanometers, the nanobubbles at the growth front of the large bubbles were swallowed by the large bubbles due to Ostwald ripening. As shown in Figure 4b, the number density of the bubbles around the large pores is much smaller than that formed at the early stage of the foaming (shown in Figure $2 \mathrm{a}$ and $2 b$ ).

Figure 5 shows the number density of bubbles for various sizes at various areas around the large bubbles. Here the area around the large bubble is illustrated in the inset of Figure 5, i.e., area $\mathrm{A}$ is located at the distance within $500 \mathrm{~nm}$ from the

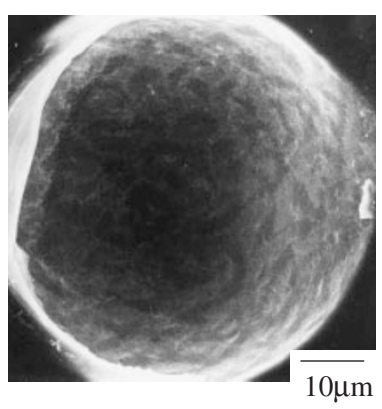

(a)



(b)
Figure 4. FE-SEM micrographs of pore structure of polycarbonate obtained after annealing the $\mathrm{CO}_{2}$-saturated specimen at $140^{\circ} \mathrm{C}$ for $200 \mathrm{~s}$ : (a) large pore, (b) exterior of large pore.



(a)

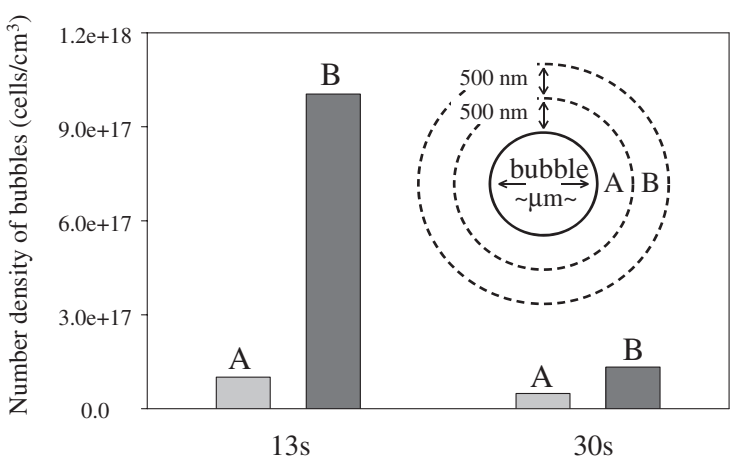

(b)

Figure 5. Number density of bubbles at various areas around the large bubbles obtained after annealing the $\mathrm{CO}_{2}$-saturated polycarbonate at $140^{\circ} \mathrm{C}$ for various annealing times: (a) bubbles having a size of several tens of nanometers, (b) bubbles having a size of several hundreds of nanometers.

growth front of the large bubble, and area B is located at the distance from $500 \mathrm{~nm}$ to $1000 \mathrm{~nm}$ from the growth front. The number density of bubbles in the area $\mathrm{A}$ is smaller than that in the area $\mathrm{B}$, indicating that the number density of nanobubbles is smaller as the distance from the growth front of the large bubble is shorter. As the large bubble grows with increasing the annealing time, the number density of nanobabbles around the large bubbles becomes smaller for both nanobubbles having a size of several ten and hundred nanometers, suggesting that larger nanobubbles having a size of several hundred nano- 


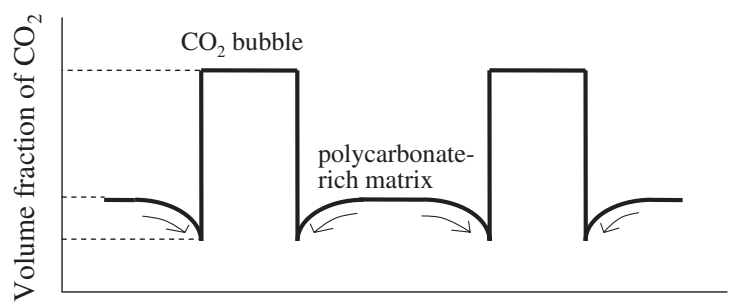

Distance

Figure 6. Schematic illustration of concentration gradient formed near the growth front of large $\mathrm{CO}_{2}$ bubble because of a diffusion-controlled process of nucleation and growth.

meters also disappear when they exist near the growth front of the large bubbles of micrometer scale. These results might support the explanation for the disappearance of the nanobubbles by Ostwald ripening due to the evaporation-condensation mechanism by the existence of large bubbles. The nucleation and growth process of foaming is explained by the diffusion-induced growth of a spherical gas bubble. Because of the diffusion-controlled process, a concentration gradient is formed near the growth front of a large bubble. ${ }^{6,7,15-19}$ The $\mathrm{CO}_{2}$ concentration in the polymer matrix decreases as the distance from the large bubbles decreases, as schematically shown in Figure 6. The low $\mathrm{CO}_{2}$ concentration near the growth front of the large bubble and the diffusion of $\mathrm{CO}_{2}$ from the polycarbonate-rich matrix due to the diffusion-controlled process may enhance Ostwald ripening.

\section{CONCLUSION}

FE-SEM observation revealed that nanobubbles smaller than a critical bubble size could be frozen by rapid cooling of $\mathrm{CO}_{2}$ saturated polycarbonate during the foaming. Because of the nucleation of new nanobubbles during the growth of the preexisting nanobubbles, the size and number of the nanobubbles increased with annealing time at an early stage of the foaming. However, most of the initially formed nanobubbles disappeared during the foaming and only a few bubbles grew to be larger ones of several tens of micrometers. The disappear- ance of the small nanobubbles might be attributed to coalescence and Ostwald ripening: large bubbles are obtained by the coalescence of neighboring nanobubbles and then they grow at the expense of smaller ones due to the evaporationcondensation mechanism. Such structural development is helpful for understanding the nucleation and growth process for new phase formation.

Received: September 14, 2007 Accepted: December 29, 2007 Published: February 19, 2008

\section{REFERENCES}

1. J. W. Gibbs, "The Scientific Papers: Vol. 1, Thermodynamics," Dover Publications, New York, 1961.

2. "Foam Extrusion-Principles and Practice," S.-T. Lee, Ed., CRC Press, Boca Raton, 2000, p 81.

3. O. Olabisi, L. M. Robeson, and M. T. Shaw, "Polymer-Polymer Miscibility," Academic Press, New York, 1979.

4. I. W. Hamley, "Introduction to Soft Matter," John Wiley \& Sons, Ltd., Chichester, 2000.

5. G. Strobl, "The Physics of Polymers: Concepts for Understanding Their Structures and Behavior," 3rd ed., Springer, Berlin, 2007.

6. M. Hatanaka and H. Saito, Macromolecules, 37, 7358 (2004).

7. M. A. Shafi, J. G. Lee, and R. W. Flumerfelt, Polym. Eng. Sci., 36, 1950 (1996).

8. M. Ohshima, "Polymeric Faming Simulation: Batch and Continuous," S.-T. Lee, Ed., CRC Press, Bora Raton, 2000, p 145.

9. L. Mandelkern, "Crystallization of Polymers," 2nd ed., Vol. 2, Cambridge University Press, Cambridge, 2004.

10. J. S. Colton and N. P. Suh, Polym. Eng. Sci., 27, 500 (1987).

11. S. K. Goel and E. J. Beckman, Polym. Eng. Sci., 34, 1137 (1994).

12. W. Ostwald, Z. Phys. Chem., 34, 495 (1900).

13. T. Ohta, Prog. Theor. Phys. Suppl., 79, 141 (1984).

14. L. Ratke and P. W. Voorhees, "Growth and Coarsening: Ostwald Ripening in Material Processing," Springer, Berlin, 2002.

15. M. Amon and C. D. Denson, Polym. Eng. Sci., 24, 1026 (1984).

16. A. Arefmanesh, S. G. Advani, and E. E. Michaelides, Polym. Eng. Sci., 30, 1330 (1990).

17. N. S. Ramesh, D. H. Rasmussen, and G. A. Campbell, Polym. Eng. Sci., 31, 1657 (1991).

18. B. Krause, R. Mettinkhof, N. F. A. van der Vegt, and M. Wessling, Macromolecules, 34, 874 (2001).

19. D. Xu, R. Pop-Livev, C. B. Park, and R. G. Fenton, J. Cell. Plastic., 41, 519 (2005). 